\title{
PRÁCTICAS DIRECTIVAS QUE FAVORECEN EL ROL DE LOS PADRES EN LA EDUCACIÓN DE SUS HIJOS
}

\author{
LEADERSHIP PRACTICES TO FOSTER \\ PARENTAL ROLE IN \\ CHILDREN'S EDUCATION
}

\author{
Francisco Larraín ${ }^{1}$ \\ https: / / orcid.org/0000-0003-3679-3375 \\ Joaquín León ${ }^{2}$ \\ https: / / orcid.org/ 0000-0003-2698-3511
}

Recibido: enero 1, 2020 - Aceptado: abril 29, 2020.

\section{RESUMEN}

El compromiso de la familia con la escuela favorece el aprendizaje de los niños. El propósito de esta investigación es identificar los factores mediadores que, en el liderazgo escolar, pueden mejorar la colaboración de la familia y la escuela. Para ello, realizamos una revisión sistemática de la literatura para identificar patrones y formas a través de las cuales el liderazgo escolar puede influir en el compromiso de los padres con sus hijos y con la escuela. Encontramos diversas actividades y funciones de liderazgo, así como distintos niveles en la investigación del tema entre países hispanoamericanos y anglosajones. Se concluye que es necesario expandir y profundizar la investigación de variables relativas a la familia en Hispanoamérica, con foco en las relaciones entre la familia y la escuela que favorezcan el desempeño de los padres, más que en las actividades en las que la familia se involucra.

Palabras clave: Liderazgo escolar, crianza del niño, relación familia escuela, variables relativas a la familia.

\footnotetext{
Doctor en Educación (EdD), Universidad de Pennsylvania. Profesor investigador, Liderazgo escolar y liderazgo distribuido, Centro de Investigación en Educación y Aprendizaje, Universidad de los Andes, Santiago, Chile. flarrain@uandes.cl

2 Máster (c) Matrimonio y Familia, Universidad de Navarra. Abogado, Profesor de Historia y Ciencias Sociales, Ayudante de Investigación, Universidad de los Andes, Santiago, Chile. jleon1@miuandes.cl
} 


\begin{abstract}
The school- family relationship fosters student engagement and learning. The purpose of this literature review is to identify factors in school leadership that might influence and improve family-school collaboration. We did a systematic literature review that allows us to identify patterns and different ways that have proven effective for school leaders to gain influence in parents' engagement with their children. We found a repertoire of activities and leadership functions that could be applied. Also found, different research levels and approaches between Hispano-American and Anglo-Saxon Samples. We concluded that it is necessary to expand and go further into family- related variables in Hispano-American, focusing on the relationship between the school and family, rather than activities where the family gets involved.
\end{abstract}

Keywords: School leadership, Parenting, School family relationship, Family-related variables.

\title{
INTRODUCCIÓN
}

La formación de las nuevas generaciones ha sido, y siempre será, una preocupación constante para toda la sociedad. Esta inquietud se ha extendido en los diversos países latinoamericanos, posicionando a la educación como uno de los mayores focos de interés para los gobiernos y entidades civiles. Si bien existen múltiples sistemas y enfoques educativos, hay un gran consenso sobre la importancia del rol que desempeña la familia en la educación de sus hijos (Goodall, 2018; Heath, Maghrabi y Carr, 2015; Hoover-Dempsey, Walker, Sandler, Whetsel, Green, Wilkins y Closson, 2005; Selwyn, Banaji, Hadjithoma-Garstka y Clark, 2011) y en las posibilidades que ofrece el liderazgo ejercido en los centros educativos para favorecer las condiciones en que los estudiantes aprenden (Goodall, 2018; Hoover-Dempsey et al., 2005; Leithwood, Harris y Hopkins, 2019).

Hasta ahora, la influencia del liderazgo escolar ha sido estudiada mayoritariamente sobre el ejercicio o la práctica docente (Anderson, 2010; Bernal e Ibarrola, 2015; Bolívar, 2010; Leithwood et al., 2010; Villa 2015; Volante, Valenzuela, Díaz, Fernández y Mladinic, 2018), quedando la familia y la práctica de los padres en un plano relevante, pero menos estudiado. En el primer caso, los líderes escolares pueden influir en la enseñanza y aprendizaje promoviendo el desarrollo de las capacidades, la motivación y las condiciones de trabajo de los docentes (Anderson, 2010; Leithwood y Riehl, 2005); mientras que para el caso de las familias, particularmente para la práctica de los padres, existe menos evidencia de los factores y las actividades en las cuales los líderes escolares pueden influir para favorecer el desempeño de los padres y apoderados, en favor del aprendizaje y desarrollo socioemocional de los estudiantes. Luego, para lograr que los estudiantes alcancen su desarrollo intelectual y humano, el liderazgo escolar podría focalizar su esfuerzo en movilizar varios factores y actividades que influyen y afectan -positiva o negativamente- el proceso educativo de los alumnos en el hogar.

Este trabajo es una revisión sistemática de la literatura, cuyo propósito es explorar aquellos factores y aquellas actividades que los líderes educativos pueden modificar para influir en el desempeño de los padres, considerando que aún es necesario conocer cuáles podrían ser algunos de estos factores y actividades sobre los que se ha escrito en la literatura. La pregunta principal que guía esta revisión sistemática de la literatura es: ¿cuáles son las actividades y los factores sugeridos, acerca de la colaboración entre la familia y la escuela, a través de los que el liderazgo escolar puede influir en la práctica educativa de los padres? 


\section{MARCO TEÓRICO}

El rol de la familia es uno de los factores fundamentales que, junto con el establecimiento educacional, promueven directamente el aprendizaje y desarrollo socioemocional de los estudiantes. Leithwood, Patten y Jantzi (2010) indican que, de las cuatro variables que afectan el aprendizaje de los estudiantes, la que más influye es la familia, aunque consideran que se sabe poco sobre cómo el establecimiento escolar puede apoyar dicha labor.

Las investigaciones referidas a la participación de los padres señalan dos grandes resultados: el decaimiento paulatino de su participación a medida que los estudiantes avanzan en los niveles escolares (Simon, 2004) que, según las estimaciones de los directores, puede disminuir hasta un $36 \%$ en la educación secundaria (Flynn y Nolan, 2008, p. 9); y el impacto que tiene el nivel de participación de los padres en la educación de sus hijos. Respecto de este último, Hattie (2017) señala que algunas de las variables familiares que pueden causar mayor impacto en el aprendizaje escolar de los estudiantes son las dinámicas positivas del hogar y la familia (0.52), el involucramiento de los padres (0.5) e intervenciones tempranas $(0.42)$.

Si la familia proporciona el entorno educativo principal de un niño, y la participación de los padres en la educación formal de sus hijos mejora el rendimiento de estos últimos (Epstein, 2010; Leithwood et al., 2010; Razeto, 2016; Rivas y Ugarte, 2014; Valdés y Sánchez, 2015), apoyar y crear los espacios para que los padres puedan criar a sus hijos adecuadamente puede ser uno de los aspectos más relevantes en el ejercicio del liderazgo. Consecuentemente, Epstein (2010) reafirma el rol fundamental de las familias en la educación de los niños y adolescentes, afirmando que «la forma en que los colegios se preocupan por sus alumnos se refleja en la forma que se preocupan por la familia de sus estos» (p. 81). Luego, no se trata solamente de ocuparse del aprendizaje de los alumnos, sino también de los padres, ayudándoles a involucrarse como primeros y principales educadores en la educación de sus hijos. Esto es compartido por Rivas y Ugarte (2014) quienes indican que «la participación de las familias en la escuela tiene un efecto positivo y duradero en el desarrollo del niño en el plano académico, social y motivacional» (p. 155). En la misma línea, concluye Razeto (2016) que las «familias más involucradas con la escuela y más comprometidas en la educación, tienen niños con un mejor desempeño que aquellas familias que no lo están» (p. 21).

\subsection{Colaboración familia-escuela}

Entendemos por colaboración entre la familia y la escuela, al esfuerzo conjunto para educar a los hijos de los primeros, que a su vez son estudiantes de los segundos (Epstein, 2010). No se trata solo de una forma de relacionarse o la manera por la que se comunican, sino de las actividades intencionales que realiza cada cual en su ámbito para promover el desarrollo de capacidades del estudiante frente al aprendizaje (Simon, 2004). La pregunta de este estudio sugiere que existe un ámbito sobre el cual el liderazgo escolar puede actuar para lograr que el compromiso de los padres y su desempeño como primeros educadores, favorezca la disposición y en general las condiciones de los estudiantes para aprender, haciendo que dicha colaboración sea más fructífera para alcanzar los resultados esperados.

El punto anterior nos presenta un desafío esencial para la tarea educativa de los padres, como también para el rol de los líderes escolares, ya que los padres no solo ayudan para 
desarrollar habilidades de aprendizaje en sus hijos, sino que buscan que sean buenas personas, es decir, que adquieran una capacidad que les permita obrar bien en todos los ámbitos de la existencia humana (García Hoz, 1994; Gooddall, 2018).

La educación escolar -que entendemos como un servicio a las familias- se orienta al aprendizaje técnico, intelectual y socioemocional (Leithwood et al., 2019), lo que ayuda a los estudiantes para aprender a hacer, y a la vez a ser buenas personas. En este sentido, un liderazgo escolar orientado a que los estudiantes sean buenos profesionales y buenas personas, debe ocuparse de que estos crezcan en los ámbitos en los que se desarrollan estas capacidades, más aún en las circunstancias en las que haga falta apoyar esta tarea educativa de los padres y familias de los educandos.

\subsection{Condiciones favorables para involucrar a los padres con el colegio}

El hecho de que los padres sean los primeros y principales educadores nos lleva a preguntarnos por la práctica educativa que realizan y las condiciones favorables para desarrollar esta labor. Esta práctica educativa puede ser guiada y acompañada por el colegio y sus profesores, a quienes literalmente se confía la educación de un grupo de alumnos.

Ya hemos dicho que el colegio debe involucrar a los padres para que estos jueguen un rol primario en la educación de sus hijos, pero cabe preguntarse: ¿qué prioridad tiene la familia para los colegios preocupados principalmente del aprendizaje de sus alumnos? Sabemos que el aprendizaje escolar cubre casi la totalidad de los esfuerzos que directivos y profesores realizan en un colegio, pero la literatura considera que la atención a la familia y los padres también es esencial. En cuanto a esta relación de los padres con el colegio, Epstein señala que existen seis tipos del prácticas que fomentan la participación de la familia en la escuela: Parenting (crianza de los hijos), Communicating (comunicación), Volunteering (voluntariado), Learning at Home (aprendiendo en casa), Decision Making (toma de decisiones) y Collaborating with Community (colaborando con la comunidad) (Epstein, 2010). Estas prácticas buscan vincular a los padres con la educación de los hijos, y cada una tiene algunas actividades, desafíos y resultados esperados, que involucran a padres, profesores y alumnos, y dan como resultado padres más implicados en el colegio de sus hijos y, como consecuencia, alumnos que alcanzan mejores niveles de educación (Epstein, 2010). Epstein concluye que estas prácticas y programas deben desarrollarse a largo plazo, siendo fundamental la formación del profesorado para relacionarse con la familia de los alumnos (Epstein, 2010). En este estudio nos preguntamos sobre el lugar que ocupa el liderazgo en la práctica educativa de los padres, lo que se refiere más a la crianza de los hijos o el ambiente favorable al aprendizaje en casa.

\subsection{Liderazgo escolar y prioridad en la familia}

Anderson (2010) señala que «hay que pensar el liderazgo directivo en las escuelas de manera más distribuida y colaborativa» (p. 37). La misma línea siguen Bernal e Ibarrola (2015), al afirmar que «el director no puede ser, en la mayoría de los casos, un "superagente" de la instrucción. En los estudios sobre el liderazgo distribuido, aparece más definido el papel del profesor, quien -como experto- hace de mentor de otros profesores y puede adoptar decisiones sobre la enseñanza» (p. 59). Por tanto, el liderazgo ejercido por los directivos puede desempeñar una buena influencia sobre los profesores para que 
estos, a través del contacto con las familias, involucren a los padres en el colegio para que incidan en la educación de sus hijos.

En muchas oportunidades, los docentes no poseen las capacidades de liderazgo desarrolladas y tampoco tienen las capacidades y la experiencia para trabajar con las familias y las comunidades, lo que influye en su motivación frente al tema (Pink, 2011). Según la opinión de los directores de los condados de Nassau y Suffolk, Nueva York, participantes en el estudio de Flynn y Nolan (2008, p. 10), el porcentaje de docentes que carecen de habilidades para trabajar con los padres puede llegar hasta un $77 \%$ en el primer ciclo básico, y hasta un $98 \%$ en la media.

Para desarrollar dichas capacidades y habilidades en los profesores, la perspectiva distribuida del liderazgo ofrece una oportunidad a través de la cual los directivos apoyan el desarrollo de sus profesores, más allá de la jerarquía establecida, a partir de su capacidad, motivación y experiencia con las familias (DeFlaminis, 2013). Luego, si los directivos logran formar a los docentes podrá cubrirse una mayor red de interacciones con los padres. Es así como «la labor del docente, como tutor de un grupo de alumnos y orientador de sus familias, adquiere un protagonismo relevante» (Cano y Casado, 2015, p. 17). Esto nos indica que no solo es esencial formar a los profesores y fomentar un tipo de liderazgo que favorezca la participación de docentes más allá del aula, sino también hacia otros docentes, en los padres y los demás miembros de la comunidad educativa (Bernal e Ibarrola, 2015).

\section{METODOLOGÍA}

Este trabajo conceptual es una revisión sistemática de la literatura, método utilizado para «ofrecer al lector una narración actualizada de lo que se conoce sobre un tema dado [...] para entregar ideas "frescas" para progresar en el conocimiento» (Galvan, 2017 , p. 7). Nuestro estudio examina variables relativas a la familia y las posibles actividades o áreas en que el liderazgo escolar puede influir en ellas, particularmente en la práctica educativa de los padres. La metodología sigue la guía propuesta por Galvan (2017) y la de otros artículos relevantes que han realizado revisiones sistemáticas de la literatura en el ámbito del liderazgo escolar (Hallinger, 2013; Hammad y Hallinger, 2017; Murphy, 2008).

La búsqueda se realizó en las siguientes bases de datos o motores de búsquedas: ERIC, EBSCO, Web of Science, Sage Journals Online, Springer link, Taylor and Francis Journal, JSTOR, Scopus y Google Académico. Se eligieron estos buscadores porque se especializan en educación y siguen los criterios de Hammad y Hallinger (2017, p. 4), cuya selección de fuentes se basó en que sean de fácil acceso para archivos digitales, tengan claros límites de búsquedas y garanticen la identificación de artículos que cumplen con estándares consistentes de calidad.

En las bases de datos empleamos combinaciones de palabras en idioma inglés y español, buscando traducir literalmente las búsquedas de un idioma al otro y, en caso de no arrojar resultados, agregamos sinónimos o conceptos que pudieran reemplazar la traducción literal. Además, limitamos el tiempo de publicación de nuestras búsquedas desde el año 2009 hasta el año 2019.

Inicialmente, realizamos una búsqueda general asociando conceptos como «liderazgo escolar» con «school family partnership», asociando luego el primero con «school 
family comunnication» y «family related variables» respectivamente. Después, en español, asociamos «liderazgo escolar» con «comunicación familia escuela», siguiendo la asociación de «liderazgo escolar» con «variables familia escuela» y «relación familia escuela» correspondientemente. Como resultado de estas búsquedas, descubrimos una gran cantidad de libros, artículos académicos de las diversas metodologías de investigación, tesis doctorales, trabajos de fin de grado y máster. Además observamos que existían otros conceptos relevantes para nuestro estudio, por lo que ampliamos la búsqueda en ambos idiomas combinando los conceptos «school leadership» con "parenting», «parent involvement» $\mathrm{y}$ «parent engagement», con sus respectivas traducciones «liderazgo escolar», «involucramiento parental»y con «compromiso parental».

Después agrupamos las diversas fuentes en dos grupos, de acuerdo con el idioma en que estaban escritos, seleccionando aquellos que cumplieran con los siguientes criterios de exclusión: reconocidos artículos académicos revisados por pares, relevantes para nuestra investigación, enfocados en el ámbito escolar; y excluimos aquellos que estuvieran en las siguientes categorías: libros, tesis doctorales o trabajos de fin de grado y máster. Aquellos artículos que cumplían directamente con los criterios de inclusión fueron escogidos, mientras que algunos de los trabajos que parecieran no cumplir con todos los criterios, fueron seleccionados como "posibles», para una revisión más profunda por su relevancia para el estudio.

Posteriormente leímos y analizamos la literatura, siguiendo los siguientes pasos:

1. Después de un período inicial de lectura, seleccionamos los artículos definitivos que serían analizados con detalle.

2. Realizamos la lectura de algunos artículos empleando como códigos los conceptos que empleamos para buscar los artículos, y luego fuimos levantando nuevos códigos basados en los modelos de influencia de liderazgo (Leithwood, et al., 2010) y de involucramiento parental de Epstein (2010), para precisar el levantamiento de información relativo al estudio.

3. A continuación, utilizamos el programa Atlas.ti para codificar los artículos seleccionados, con los códigos respectivos para cada grupo.

4. Siguiendo el método realizado por Murphy (2008), codificamos frases lo más cortas posibles, limitando la extensión de cada codificación a la extensión de un párrafo.

5. Luego de codificar los artículos se exportaron todas las citas a Excel, preparando ambas bases de datos para el análisis de las citas recolectadas. Obtuvimos 206 citas en inglés y 146 en español.

6. A lo largo de todo el trabajo, fuimos recordando las ideas que nos llevaron a plantearnos el artículo y la pregunta que terminó fundamentando esta investigación.

7. El análisis lo realizamos agrupando las frases por código, cotejando lo encontrado en ambos idiomas. En este análisis buscamos identificar definiciones, datos, características o tipología de conceptos, patrones y tendencias; comparamos similitudes diferencias y contradicciones entre los artículos y entre los patrones hallados; y recolectamos las frases que contenían un valor singular, respecto de los otros elementos. 
8. Mientras íbamos revisando las frases codificadas, fuimos anotando todo lo anterior, realizando conexiones que emergieron del análisis a través de los artículos.

9. A continuación, observamos los temas que surgieron de la literatura para responder a nuestra pregunta de investigación y escribimos la siguiente sección.

\section{RESULTADOS}

Los documentos encontrados inicialmente en la búsqueda de la literatura fueron 255 en español y 744 en inglés, de los cuales, como se puede observar en la figura 1, los artículos que finalmente se seleccionaron mediante la revisión sistemática de la literatura para responder a las preguntas de investigación fueron 21, de ellos, 14 están escritos en inglés y 7 en español, tal como se describe en el siguiente gráfico.

Figura 1. Diseño de revisión sistemática de la literatura
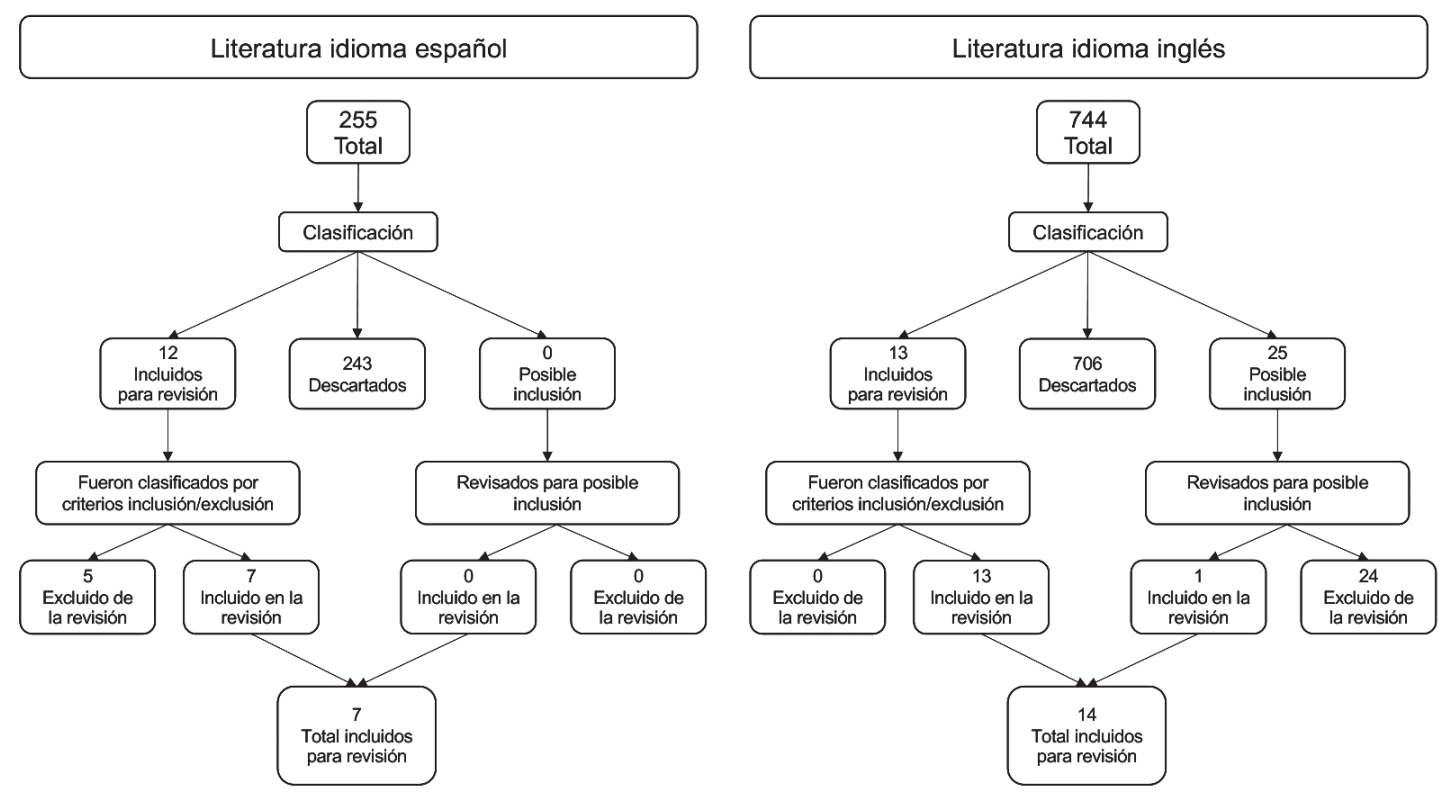

Fuente: Elaboración propia.

La tabla 1 muestra los artículos seleccionados por autor, año de su publicación, procedencia por continente y revista en la que se publicó; podemos evidenciar que predominan las investigaciones en Estados Unidos e Inglaterra. Los países de habla hispana tienen pocas publicaciones sobre el tema. 
Tabla 1: Distribución de artículos seleccionados por fecha y procedencia

\begin{tabular}{|c|c|c|}
\hline Autor (fecha de publicación) & Revista & País de publicación \\
\hline \multicolumn{3}{|l|}{ Revistas en idioma español } \\
\hline Borrell y Artal, (2014) & Estudios sobre educación & España \\
\hline Cabello y Miranda, (2016) & Revista de Evaluación de Programas y Políticas Públicas & España \\
\hline Garreta Bochaca, (2016) & Perspectiva Educacional, Formación de profesores & Chile \\
\hline Eichin y Beach, (2018) & Revista Complutense de Educación & España \\
\hline Miranda Carvajal y Castillo Armijo, (2018) & Estudios pedagógicos & Chile \\
\hline Palomeque García, y López Rodríguez (2018) & Universidad de Cienfuegos & Cuba \\
\hline Garcia et al. (2019) & Espiral. Cuadernos del Profesorado & España \\
\hline \multicolumn{3}{|l|}{ Revistas en idioma inglés } \\
\hline Warren et al. (2009) & Teachers college record & United States \\
\hline Leithwood et al. (2010) & Educational Administration Quarterly & United States \\
\hline Bryan y Griffin (2010) & School Leadership \& Management & United Kindom \\
\hline Selwyn et al. (2011) & Journal of Computer Assisted Learning & United Kindom \\
\hline Bempechat y Shernoff (2012) & Springer & United States \\
\hline Goodall, (2013) & School Leadership \& Management & United Kindom \\
\hline Barr, y Saltmarsh (2014) & Educational Management Administration \& Leadership & United Kindom \\
\hline Heath et al. (2015) & Journal of Information Technology Education & United States \\
\hline Zhao et al. (2017) & International Journal of Research Studies in Education & Philippines \\
\hline Erol \& Turhan (2018) & Educational Sciences: Theory and Practice & Spain \\
\hline Goodall (2018) & School Leadership \& Management & United Kindom \\
\hline Badri et al. (2018) & International Journal of Child Care and Education Policy & Korea \\
\hline Leithwood et al. (2019) & School Leadership \& Management & United Kindom \\
\hline Malluhi y Alomran (2019) & International Journal of Emerging Technologies in Learning & Germany \\
\hline
\end{tabular}

Fuente: Elaboración propia.

Cada artículo incluido en este estudio se revisó metódicamente en la búsqueda de citas que inicialmente se relacionaron con los descriptores usados para encontrar los artículos. En una segunda revisión, consensuamos los códigos para identificar aquellas citas que sirvieron para obtener los resultados del estudio.

Los códigos que consiguieron mayor cantidad de citas relacionadas fueron los asociados a la comunicación y/o relación familia escuela, relación entre padres e hijos, y variables relativas a la familia.

Todos los estudios indican que existe consenso sobre la importancia del rol de los padres en el desarrollo y desempeño académico escolar de sus hijos (Cabello y Miranda, 2016; Eichin y Volante, 2018; García, Santos, Alcalá del Olmo e Isequilla, 2019; Garreta, 2016; Goodall, 2018; Heath et al., 2015; Miranda y Castillo, 2018; Rivas y Ugarte, 2014; Selwyn et al., 2011). A esto podemos agregar que un estudio cuantitativo realizado en escuelas de Abu Dabhi muestra que el efecto del hogar sobre la felicidad de los hijos (0.55) puede ser mayor (0.6751) cuando se agrega el efecto indirecto que los padres también ejercen indirectamente a través de la escuela (Badri, Al Nuaimi, Guang, Al Sheryani y Rashedi, 2018, p. 8). Por su parte, el estudio de Miranda y Castillo (2018) establece que el apoyo y participación familiar en los procesos educativos son influenciados por la comunicación de los padres con la escuela (0.85), conocimiento de la escuela (0.83), y apoyo y supervisión del aprendizaje en el hogar (0.76). También se evidencia que el desarrollo psicosocial y académico de los estudiantes puede perjudicarse si existen conflictos entre sus profesores y padres (Bempechat y Shernoff, 2012). 
Sin embargo, aparece una preocupación transversal entre los estudios, referida a la baja participación, involucramiento y compromiso de los padres con sus escuelas, lo que representa a su vez una gran oportunidad para las escuelas. Adicionalmente, también es relevante el rol de los directivos y líderes escolares para dar forma al desempeño de los padres en relación con el establecimiento (Barr y Saltmarsh, 2014). De esta revisión se desprende la necesidad de apoyar las variables relativas a mejorar el desempeño y participación de los padres como una de las principales tareas para el liderazgo escolar.

\section{1 Áreas y actividades del desempeño de los padres en las que influye el liderazgo escolar}

El desempeño de los padres, tanto en el hogar como en la escuela, es un concepto que utilizamos para resumir aquello que los autores definen como involucramiento o compromiso parental, entre otros, los que se refiere a las actividades que realizan los padres para favorecer el crecimiento de sus hijos en su hogar como en la escuela (Barr y Saltmarsh, 2014; Malluhi y Alomran, 2019). Estas actividades son, en su mayoría, factores alterables que afectan el desempeño de los padres y que, por lo tanto, influyen sustancialmente en el desarrollo cognitivo, social y emocional de los estudiantes (Leithwood et al., 2019).

La tabla 2 muestra las actividades señaladas en los artículos, organizadas por categorías o funciones directivas para facilitar, siguiendo el modelo de Leithwood et al., (2008), la comprensión sobre cómo el liderazgo escolar podría influir en el desempeño de los padres.

Tabla 2. Actividades directivas agrupadas según funciones sugeridas por Leithwood et al. (2010) para influir en el desempeño de los padres directa 0 indirectamente a través de los docentes

\begin{tabular}{|c|c|c|}
\hline Función directiva & Actividades & \\
\hline Mostrar la dirección & $\begin{array}{l}\text { Crear una visión compartida } \\
\text { Compartir información y comentar decisiones } \\
\text { Promover altas expectativas de los padres y profesores sobre los estudiantes } \\
\text { Orientar sobre la necesidad de trabajar colaborativamente }\end{array}$ & 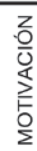 \\
\hline Desarrollo Profesional/Desempeño & $\begin{array}{l}\text { Profesores y padres } \\
\text { Promover altas expectativas entre profesores y padres } \\
\text { Apoyar desarrollo socioemocional de profesores y padres } \\
\text { Profesores } \\
\text { Desarrollar capacidad comunicacional de profesores } \\
\text { Promover desarrollo de liderazgo entre profesores } \\
\text { Padres } \\
\text { Considerar factores socioeconómicos para establecer desarrollo de la familia } \\
\text { Desarrollar estrategias de comunicación padres-hijos } \\
\text { Incrementar el capital social e intelectual de los padres sobre la enseñanza escolar } \\
\text { Fomentar un tiempo significativo entre padres e hijos }\end{array}$ & 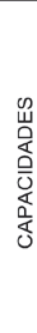 \\
\hline Desarrollo de la organización & $\begin{array}{l}\text { Seleccionar canales de comunicación adecuados } \\
\text { Promover el voluntariado entre padres y profesores } \\
\text { Promover comunidades de padres según intereses } \\
\text { Crear clima de confianza, colaboración y psicológicamente positivo en la escuela }\end{array}$ & 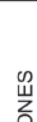 \\
\hline $\begin{array}{l}\text { Mejoras instruccionales } \\
\text { (desempeño padres) }\end{array}$ & $\begin{array}{l}\text { Apoyar y acompañar actividades de aprendizaje en casa } \\
\text { Promover ambiente de aprendizaje en casa } \\
\text { Compartir criterios de comportamientos en el hogar (uso de pantallas) } \\
\text { Educar en el uso de equipos tecnológicos al servicio del aprendizaje }\end{array}$ & 号 \\
\hline
\end{tabular}

Fuente: Elaboración propia. 
En la tabla 2 y siguiendo el modelo propuesto por Leithwood et al. (2010), se indican las actividades para influir en el desempeño de los padres de manera directa o indirecta a través de los docentes. Del mismo modo, se muestra cómo el liderazgo escolar influye indirectamente en el aprendizaje de los estudiantes a través del desempeño de los profesores y padres del estudiante.

Se puede observar que las áreas o actividades de desempeño de los padres, sobre las cuales el liderazgo escolar podría influir, han sido estudiadas de manera más diversa en las investigaciones de origen anglosajón, donde el análisis ha evolucionado desde los estudios enfocados a las actividades en las que participan los padres (e.g. leer libros a los hijos o apoyar sus tareas en la casa) hacia otros focalizados en profundizar sobre las relaciones (e.g. la actitud de los padres frente al aprendizaje o las expectativas sobre los logros) que incrementan la motivación, como fundamento del desempeño de los padres en el hogar (Bempechat y Shernoff, 2012; Goodall, 2018; Warren, Hong, Rubin, y Uy, 2009). Según esto, actividades como apoyar las tareas de los hijos son también oportunidades para que los padres transfieran a sus hijos habilidades, experiencias y estrategias, por ejemplo, de la administración del tiempo y la resolución de problemas (Bempechat y Shernoff, 2012).

La asimetría que se observa en el cuadro 2 desafía la investigación en español para que avance en el desarrollo de este segundo enfoque hallado en la literatura anglosajona, sobre todo entendiendo que todo lo que pueda hacerse para mejorar los factores que influyen en el estudiante fuera de la escuela podría transformarse en palancas muy significativas para el liderazgo escolar (Leithwood et al., 2019).

\subsection{Liderazgo escolar y desempeño de los padres}

Una de las temáticas que emerge como patrón en la revisión de los artículos señalados es la identificación de los líderes o agentes que influyen en el desempeño de los padres (Erol y Turhan, 2018; Garreta, 2016; Goodall, 2018; Leithwood et al., 2019; Leithwood et al., 2010; Rivas y Ugarte, 2014). Coherentemente con la evolución que ha tenido el liderazgo en los últimos años, y particularmente en la literatura acerca del liderazgo distribuido, la influencia en el desempeño de los padres no se trata solamente de un rol directivo, sino también de profesores, consejeros, tutores (Cabello y Miranda, 2016; García et al., 2019), u otra persona que ya sea por encargo, o por su compromiso y capacidades frente al tema (Leithwood et al., 2019; Miranda y Castillo, 2018), asume o debería asumir la tarea de liderar la relación de la escuela con la familia.

Este escenario del liderazgo distribuido -la figura de los consejeros o agentes culturales (Bryan y Griffin, 2010), encargados de las familias, tutores, profesores e incluso miembros de la comunidad, puede aportar en la tarea de apoyo a la labor de los padres (Malluhi y Alomran, 2019)-, aparece como una aproximación adecuada para que la organización aborde su relación con las familias (Malluhi y Alomran, 2019; Rivas y Ugarte, 2014). Todos estos agentes asumen roles de liderazgo a través del cual pueden apoyar mejoras en el desempeño de los padres para educar a sus hijos. El enfoque del liderazgo distribuido se presenta como una atractiva aproximación para apoyar a los padres, porque su foco de interés son las interacciones (Spillane, 2006) en circunstancias en que, para incrementar el compromiso de los padres, se necesiten fortalecer las relaciones dentro de la comunidad (padres y profesores), «resaltando el desarrollo de [dichas] relaciones, desarrollando las capacidades de los padres [y profesores] como líderes y orientando su tarea para estrechar las diferencias de cultura y poder entre padres y profesores» (Warren et al., 2009, p. 2239). 
Otro aspecto que se considera para el diseño del liderazgo es que las particularidades y la singularidad en la realidad de cada escuela, exige que el desarrollo profesional y la búsqueda de mejoras en las capacidades de los líderes escolares debe realizarse en función de cada contexto en el que se desenvuelven las familias (Rivas y Ugarte, 2014). Si bien la mayoría de los autores enfatizan que la influencia de la familia en los logros de los estudiantes no depende de su condición sociodemográfica, sí se considera necesario que cada escuela sepa identificar sus propias problemáticas para abordar las carencias y brechas particulares que tenga (Leithwood et al., 2019).

Las expectativas de los directivos son un predictor significativo para el desempeño de los profesores y demás representantes del colegio que se relacionan con la familia (Bryan y Griffin, 2010).

\subsection{Limitaciones para el liderazgo escolar}

En los artículos revisados se hallaron patrones sobre las limitaciones que enfrentan los líderes escolares para apoyar el desempeño de los padres. Entre los principales que encontramos se encuentran los siguientes:

\section{- Tiempo}

Esta limitación se refiere a la estrechez y escasez de tiempo, y a las diferencias de horario disponible que restringe la relación entre padres y educadores (García et al., 2019; Goodall, 2018; Malluhi y Alomran, 2019; Rivas y Ugarte, 2014; Selwyn et al., 2011). Los padres tienen disponibilidad de tiempo fuera del horario escolar y los profesores no disponen de ese espacio para trabajar con los padres. En razón de lo anterior, tanto padres como profesores cuentan con poco tiempo y en distintos horarios para encontrarse (García et al., 2019), hecho que dificulta la relación y comunicación entre ellos.

Entre las oportunidades que los líderes escolares pueden movilizar para abordar las dificultades de tiempo, se encuentran: a) reconocer y mostrar el poder de los padres para negociar el tiempo destinado hacia ellos (Bryan y Griffin, 2010); b) crear una red de apoyo de padres y otros líderes de la escuela para extender la tarea en los espacios en que no es posible asistir (Bryan y Griffin, 2010; Malluhi y Alomran, 2019), y c) un adecuado uso de tecnología para aumentar la eficiencia y eficacia de diversos canales de comunicación entre la escuela y los padres (Chrispeels, 1996; Garreta, 2016; García et al., 2019; Heath et al., 2015).

\section{- Capacidad y formación}

Tal vez una de las variables más relevantes, relacionadas con el desempeño de los padres, es su capacidad y formación, lo mismo que la de los profesores. La capacidad de los primeros se refiere al conocimiento y facultad que tienen para ser miembros activos en la educación de sus hijos (e.g. el saber ayudarlos a adquirir el método de estudio; el desarrollar en ellos diversas virtudes y valores; los conocimientos acerca de las características de las distintas edades, como la adolescencia, entre otros) y el empleo activo de la tecnología disponible (García et al., 2019; Heath et al., 2015; Selwyn et al., 2011). Todos los anteriores parecen afectar la noción de eficacia personal para desempeñarse, tanto para profesores como para padres (Bempechat y Shernoff, 2012; Rivas y Ugarte, 2014). La eficacia personal -aquella creencia de que uno es capaz de desempeñar bien una tarea (Bandura, 1977)- es indicada como una de las variables más influyentes en la capacidad de profesores para apoyar a los padres, y de los padres para criar a sus hijos. 
Algunas de las sugerencias para incrementar la capacidad de líderes escolares relacionadas con la familia son: a) empleo de datos como herramienta para conocer a las familias, incrementar estrategias de apoyo hacia ellas, mostrar los beneficios, los logros (Bryan y Griffin, 2010), y dimensionar las brechas; b) desarrollar altas capacidades socioemocionales y de comunicación, entre directivos y profesores para mejorar su efectividad en el trato con los padres (Barr y Saltmarsh, 2014); c) ampliar el alcance del trato con los estudiantes hacia una relación que refleje una actitud cálida, preocupada y apoyadora (Bempechat y Shernoff, 2012); d) si bien se recomienda aumentar el empleo de tecnología para abordar las limitaciones de tiempo (García et al., 2019; Heath et al., 2015; Selwyn et al., 2011), también se enfatiza el hecho de que las relaciones cara a cara, o presenciales, favorecen una forma directa de colaboración entre profesores y padres (Warren, Hong et al., 2009), y e) emplear un marco de referencia para agrupar a los profesores según su capacidad para estimular el compromiso de los padres, como por ejemplo el proyecto de apoyo y asistencia para profesores noveles de Santa Cruz, Estados Unidos, o los otros marcos sugeridos por Chrispeels (1996).

Las iniciativas anteriores buscan crear oportunidades para que padres y profesores alcancen un dominio sobre su desempeño y también sobre el rol que ejerce o desarrolla la otra parte, ya sean padres o profesores (Bryan y Griffin, 2010). Y alcanzado este dominio, tanto los padres como los profesores se sentirán más capacitados y, por ende, más motivados para participar en esta relación (entre padres y profesores) que ayude al desarrollo de cada alumno (Chrispeels, 1996).

\section{- Apertura a comprender la situación de los otros}

La relación entre profesores y padres se ve influenciada por el conocimiento de cada uno sobre la actividad del otro. Al respecto, los artículos revisados reportan que las limitaciones para el rol de los padres se relacionan con sentimientos de vulnerabilidad (Goodall, 2018) y creencias de que los profesores no se preocupan (Bempechat y Shernoff, 2012).

Algunas sugerencias para los líderes escolares que se proponen en los artículos, para incrementar el conocimiento y la comprensión de los padres son: a) construir una visión compartida, práctica que es común al liderazgo exitoso en diversos contextos (Leithwood et al., 2019), clarificando lo que implican conceptos como buena crianza y compromiso parental (Goodall, J., 2018); b) participar en reuniones directivas para posicionar el rol de los padres en la toma de decisiones (Bryan y Griffin, 2010; García et al., 2019; Palomeque García, Alfonso Moreira y López Rodríguez del Rey, 2018; Rivas y Ugarte, 2014); c) mejorar los canales de comunicación entre los padres y profesores para que estos sean accesibles, en ambos sentidos, consistentes, informales y significativos (Cabello y Miranda, 2016; Erol y Turhan, 2018; Garreta, 2016; Heath et al., 2015; Palomeque García et al., 2018; Rivas y Ugarte, 2014; Selwyn et al., 2011), y d) demostrar afecto y genuina preocupación por los niños (Bempechat y Shernoff, 2012).

\section{DISCUSIÓN}

Las variables que afectan o pertenecen al desempeño de los padres son múltiples y una clara comprensión, organización y posterior estudio sobre sus efectos en el desarrollo de los estudiantes es necesaria para la investigación sobre la influencia que puede ejercer el liderazgo escolar en la familia. 
Para el liderazgo escolar es relevante conocer, no solamente la composición de estas variables, sino también el alcance de la organización escolar para influir en ellas.

Una característica común entre los artículos, cuando se refieren a las limitaciones, es que tanto padres como profesores y otros líderes escolares - no se habla de los directores- carecen del conocimiento sobre la actividad del otro, del dominio de lo que les corresponde hacer para interactuar con los otros y, por lo tanto, se restan o no son invitados a participar en el ámbito de la escuela (cuando se refiere a los padres) o en el hogar (cuando se refiere a los representantes de la escuela). Estos tres elementos son los que Daniel Pink (2011) describe como componentes de la motivación de las personas. Por ejemplo, comprender que la acción de realizar tareas con los hijos es, a su vez, una oportunidad para transmitir experiencias de los padres sobre cómo resolver problemas o administrar el tiempo; asimismo, sirve para desarrollar conocimiento, participación y dominio sobre un aspecto, influyendo positivamente en la motivación de padres e hijos. Del mismo modo, el apoyo y la calidez de los padres en este tipo de actividades, promueven una motivación particular hacia los logros académicos, entendiendo que los conflictos y las tensiones producen también lo contrario (Bempechat y Shernoff, 2012). Este tipo de recomendaciones se pueden encontrar en la literatura hispanoamericana (Bernal, Rivas y Urpí, 2012; Lyford-Pike, 1997) pero aún es posible desarrollar investigación para evidenciar los efectos de estas actitudes en el desempeño de los padres, que también pueden ser promovidas desde el liderazgo escolar.

Consecuentemente, surge una atractiva relación para explorar la motivación de padres, profesores y estudiantes en función de estos elementos que configurarían su motivación para que los primeros apoyen el desarrollo de los estudiantes, y para que estos incrementen su disposición para aprender y relacionarse con otros, particularmente con sus hijos.

La relación entre padres y profesores se ve fuertemente limitada por las condiciones laborales y la disposición que cada uno tenga para interactuar con el otro. Los padres se encuentran limitados para participar en actividades que no sean fuera del horario de la escuela, mientras que los profesores funcionan a la inversa.

Referirse a los programas que se mencionan en la literatura anglosajona, responde a este avance de la investigación en el sentido de ofrecer criterios para desarrollar planes de mejora con orientación en el mejoramiento de la relación entre la escuela y la familia, encaminados al desempeño de los padres en el hogar (Bempechat y Shernoff, 2012; Goodall, 2018).

\section{CONCLUSIONES}

En la literatura existe consenso sobre la relevancia del papel que juegan los padres en la educación de sus hijos. Los padres son fundamentales en la educación de sus hijos y entre más se involucren, aportan a su desarrollo.

Luego, es necesario expandir y profundizar la investigación sobre las diversas actividades y / o factores en que el liderazgo escolar puede influir; sobre todo en aquellos que pudieran tener un mayor impacto en la realidad de cada establecimiento y de las familias con las que se vincula. Más aún, se requiere identificar factores y actividades cuyo efecto sea mayor en el menor tiempo posible. 
Por ejemplo, diseñar mejoras organizacionales que permitan dedicar tiempo y recursos al trabajo con los padres y apoderados; enmarcar la narrativa del establecimiento sobre la base de las altas expectativas del desempeño de los profesores, padres y estudiantes; crear un ambiente socioemocionalmente positivo, en donde el trato afectivo predomine sobre las complejidades de las interacciones entre profesores, padres, estudiantes y directivos del establecimiento. Para lo anterior, nos parece primordial ampliar la capacidad de aprender de los otros, para lo que el liderazgo basado en la evidencia -encaminado al desempeño de los padres y apoderados-, puede ser un aporte significativo en el desarrollo de una cultura orientada al servicio de las familias.

También nos parece relevante que las mismas comunidades educativas diagnostiquen sus capacidades para centrar la formación, tanto de padres como de profesores, según sus necesidades. En la literatura hay acuerdo en que los profesores y padres requieren formación, pero no existe consenso sobre qué tipo de formación demandan.

Este trabajo aspira a contribuir en esta línea para orientar los programas de formación de padres y profesores. Con este objetivo, se necesita expandir y profundizar la investigación sobre las variables, los factores o las actividades que influyen en el desarrollo del desempeño de los padres y apoderados en Hispanoamérica. Para ello, el ejemplo de la literatura anglosajona sugiere que este esfuerzo se focalice en comprender las relaciones entre la familia y la escuela, más que en las actividades en que la familia se involucra. Lo anterior significa explorar, por una parte, no solo las actividades que ayuden a que el desempeño de los padres favorezca el ejercicio académico y desarrollo humano de los estudiantes, sino en describir los significados que tanto profesores como padres tienen sobre la relación de la familia con la escuela; a partir de lo cual se pueda entender las similitudes y diferencias que expliquen los aciertos y dificultades de los establecimientos y sus familias para trabajar colaborativamente. Esto supone asimismo establecer patrones comunes entre diversas realidades sociodemográficas, a partir de los cuales se desplieguen iniciativas de perfeccionamiento profesional que sirvan a la variedad de proyectos educativos, así como también es relevante identificar aquellos aspectos que acentúan estas diferencias, para orientar a los líderes educativos sobre la necesidad de innovar en sus procesos de mejora sobre la base de las diversas particularidades de las comunidades educativas. Adicionalmente, se requiere estudiar cuáles son los factores y/o las actividades que tienen un mayor impacto en la relación del establecimiento con las familias, focalizados en el desempeño de los padres; esto exige aprender de lo que ocurre en los hogares para que, a partir de ahí, se apoye a los padres en la gran tarea de educar a sus hijos y de entregarles experiencias socioemocionales, técnicas y cognitivas que se ordenen a su mejor desempeño como estudiantes y en sus futuros roles de la sociedad. 


\section{REFERENCIAS}

Anderson, S. (2010). Liderazgo directivo: claves para una mejor escuela. Psicoperspectivas, $9(2), 34-52$.

Badri, M., Al Nuaimi, A., Guang, Y., Al Sheryani, Y. \& Al Rashedi, A. (2018). The effects of home and school on children's happiness: a structural equation model. International Journal of Child Care and Education Policy, 12(1), 17.

Barr, J. \& Saltmarsh, S. (2014). «It all comes down to the leadership»: The role of the school principal in fostering parent-school engagement. Educational Management Administration \& Leadership, 42(4), 491-505. https://doi-org.uandes.idm.oclc. org $/ 10.1177 / 1741143213502189$

Bandura, A. (1977). Self-efficacy: toward a unifying theory of behavioral change. Psychological review, 84(2), 191.

Bempechat, J. \& Shernoff, D. J. (2012). Parental influences on achievement motivation and student engagement. In Handbook of research on student engagement (pp. 315-342). Springer, Boston, MA.

Bernal Martínez de Soria, A. (2015). Liderazgo del profesor: objetivo básico de la gestión educativa. Revista Iberoamericana de Educación, (67), 55. Recuperado de https: / / ezproxy. si.unav.es:2098/login.aspx?direct=true\&AuthType=ip,url\&db=edsdnp\&AN=edsd$\underline{\text { np.5082914ART\&lang }=\text { es\&site }=\text { eds-live\&scope }=\text { site }}$

Bernal, A., Rivas S. y Urpí, C. (2012). Educación Familiar, Infancia y Adolescencia. Madrid, España: Ediciones Pirámide.

Bolívar Botía, A. (2010). El liderazgo educativo y su papel en la mejora: Una revisión actual de sus posibilidades y limitaciones. Psicoperspectivas, (2), 9. Recuperado de https: / / ezproxy.si.unav.es:2098/ login.aspx?direct=true\&AuthType=ip, url\&db=edsdnp\&AN=edsdnp.3664340ART\&lang $=$ es\&site $=$ eds-live\&scope $=$ site

Bryan, J. A. \& Griffin, D. (2010). A Multidimensional Study of School-Family-Community Partnership Involvement: School, School Counselor, and Training Factors. Professional School Counseling. Recuperado de https:/ / doi.org/10.1177/2156759X1001400108

Cabello, S. A. y Miranda, J. G. (2016). La participación de las familias en la escuela: una cuestión compleja. Revista de Evaluación de Programas y Políticas Públicas, 1(7), $28-47$.

Cano González, R. y González, M. C. (2015). Escuela y familia. Dos pilares fundamentales para unas buenas prácticas de orientación educativa a través de las escuelas de padres. Revista electrónica interuniversitaria de formación del profesorado, 18(2), 15-27.

Chrispeels, J. (1996). Evaluating Teachers' Relationships with Families: A Case Study of One District. The Elementary School Journal, 97(2), 179-200. Retrieved from http:/ / www. jstor.org.uandes.idm.oclc.org/stable/1002243

DeFlaminis, J. A. (2013). «The Implementation and Replication of the Distributed Leadership Program: More Lessons Learned and Beliefs Confirmed». Paper presented at the annual meeting of the American educational research association, San Francisco, California. 
Eichin, N. y Volante, P. (2018). Liderando alianzas entre escuelas, familias y comunidades: una revisión sistemática. Revista Complutense de Educación, 29(2), 595.

Epstein, J. L. (n.d.). School/family/community partnerships: Caring for the children we share. Phi Delta Kappan, 92(3), 66-96. Retrieved from https://ezproxy. $\underline{\text { si.unav.es:2098/login.aspx?direct=true\&AuthType }=i p, u r l \& d b=e d s e l c \& A N=e d-}$ selc.2-52.0-80051660044\&lang=es\&site=eds-live\&scope=site

Erol, Y. C. \& Turhan, M. (2018). The Relationship between Distributed Leadership and Family Involvement from Parents' Perspective. Educational Sciences: Theory and Practice, 18(3), 525-540.

Flynn, G. \& Nolan, B. (2008). What do school principals think about current school-family relationships? NASSP Bulletin, 92(3), 173-190.

Galvan, J. L. \& Galvan, M. C. (2017). Writing literature reviews: A guide for students of the social and behavioral sciences. Routledge. Devon: UK

García, L. M. P., Villalba, M. J. S., del Olmo Fernández, M. J. A., y Alarcón, E. I. (2019). El desafío educativo del siglo XXI: relevancia de la cooperación entre familia y escuela. The educational challenge of the twenty-first century: relevance of the cooperation between family and school. Espiral. Cuadernos del Profesorado, 12(24), 19-29.

García Hoz, V. (1994). La formación de la persona: puntos de referencia para su estudio. Rcuperado de https://ezproxy.si.unav.es:2098/login.aspx?direct=true\&AuthType=ip,ur-

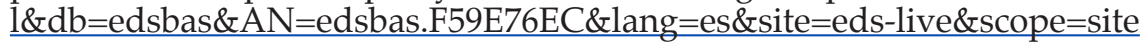

Bochaca, J. G. (2016). Fortalezas y debilidades de la participación de las familias en la escuela. Perspectiva educacional. Formación de profesores, 55(2), 141-157.

Goodall, J. (2013). Parental engagement to support children's learning: a six point model. School Leadership \& Management, 33(2), 133-150.

Goodall, J. (2018). A Toolkit for Parental Engagement: From Project to Process. School Leadership and Management, 38(2): 222-238.

Hattie, J. (2017). Visible Learning (plus), 250+ Influences on Student Achievement (Imagen) https://visible-learning.org/wp-content/uploads/2018/03/VLPLUS-252-Influences-Hattie-ranking-DEC-2017.pdf

Heath, D., Maghrabi, R. \& Carr, N. (2015). Implications of Information and Communication Technologies (ICT) for School-Home Communication. Journal of Information Technology Education, 14.

Hallinger, P. (2013). A conceptual framework for systematic reviews of research in educational leadership and management. Journal of Educational Administration, 51(2), 126149 .

Hoover-Dempsey, K. V., Walker, J. M., Sandler, H. M., Whetsel, D., Green, C. L., Wilkins, A. S. \& Closson, K. (2005). Why Do Parents Become Involved? Research Findings and Implications. The Elementary School Journal, 106(2), 105-130.

Leithwood, K., Harris, A. \& Hopkins, D. (2019). Seven strong claims about successful school leadership revisited. School Leadership \& Management, 1-18.

Leithwood, K., Harris, A. \& Hopkins, D. (2008). Seven strong claims about successful school leadership. School Leadership and Management, 28(1), $27 \mathrm{~A} 42$. 
Leithwood, K., Patten, S. \& Jantzi, D. (2010). Testing a conception of how school leadership influences student learning. Educational Administration Quarterly, 46(5), 671-706.

Leithwood, K., Harris, A. \& Hopkins, D. (2019). Seven strong claims about successful school leadership revisited. School Leadership E Management, 1-18.

Lyford-Pike, A. (1997). Ternura y firmeza con los hijos (Vol. 90). Santiago de Chile: Universidad Católica de Chile.

Malluhi, H. H. \& Alomran, N. M. (2019). Family Volunteers as Alternative Future Resources: School Leaders' Beliefs and Practices. International Journal of Emerging Technologies in Learning, 14(10).

Miranda Carvajal, C. y Castillo Armijo, P. (2018). Estudio metodológico de las variables que componen el constructo de «apoyo y participación de la familia en los procesos educativos». Estudios Pedagógicos (Valdivia), 44(1), 115-133.

Murphy, J. (2008). The place of leadership in turnaround schools: Insights from organizational recovery in the public and private sectors. Journal of Educational Administration, 46(1), 74-98.

Palomeque García, I. J., Alfonso Moreira, Y. y López Rodríguez del Rey, M. M. (2018). La participación de la familia en la institución educativa: sus condiciones en el proceso pedagógico. Universidad y Sociedad, 10(5), 398-406. Recuperado de http:/ / rus.ucf.edu. $\mathrm{cu} /$ index.php/rus

Pink, D. H. (2011). Drive: The Surprising Truth about What Motivates us. New York, NY: Riverhead Books.

Razeto, A. (2016). El involucramiento de las familias en la educación de los niños. Cuatro reflexiones para fortalecer la relación entre familias y escuelas. Páginas de Educación, 9(2), 190-216.

Rivas, S. y Ugarte, C. U. (2014). Formación docente y cultura participativa del centro educativo: claves para favorecer la participación familia-escuela. Estudios sobre educación, 27.

Simon, B. S. (2004). High school outreach and family involvement. Social Psychology of Education, 7(2), 185-209.

Selwyn, N., Banaji, S., Hadjithoma-Garstka, C. \& Clark, W. (2011). Providing a platform for parents? Exploring the nature of parental engagement with school learning platforms. Journal of Computer Assisted Learning, 27(4), 314-323.

Valdés Cuervo, Á. A. y Sánchez, P. A. (2016). Las creencias de los docentes acerca de la participación familiar en la educación. Revista electrónica de investigación educativa, 18(2), 105-115.

Volante, P., Valenzuela, S., Díaz, A., Fernández, M. \& Mladinic, A. (2019). Validation of an assessment centre process for the selection of school leaders in Chile. School Leadership \& Management, 39(1), 26-47.

Warren, M. R., Hong, S., Rubin, C. L. \& Uy, P. S. (2009). Beyond the bake sale: A community-based relational approach to parent engagement in schools. Teachers college record, 111(9), 2209-2254. 